\title{
Avaliação da qualidade de vida e percepção de mudança em pacientes com esquizofrenia
}

\author{
Assessment of quality of life and perception of \\ change in patients with schizophrenia \\ Luciana Cesari', Marina Bandeira ${ }^{1}$
}

\section{RESUMO}

Objetivo: Investigar os fatores associados à qualidade de vida (QV) em pacientes com esquizofrenia, em particular a percepção de mudanças pelo próprio paciente, em função do tratamento recebido em serviços de saúde mental. Método: $\mathrm{O}$ estudo foi conduzido em Divinópolis (MG), com pacientes atendidos no Serviço de Referência em Saúde Mental. Foram realizadas entrevistas individuais estruturadas com a aplicação da Escala de Qualidade de Vida (QLS-BR), Escala de Mudança Percebida (EMP) e questionário sociodemográfico e clínico. Foram conduzidas três análises de regressão linear múltipla, para determinar a importância relativa dos fatores preditores da QV. Resultados: Participaram deste estudo 72 pacientes, sendo a maioria do sexo masculino (59,7\%), com diagnóstico de esquizofrenia paranoide (87,5\%). A QV dos pacientes enquadrou-se na categoria de considerável prejuízo, com um

\section{Palavras-chave}

Qualidade de vida, avaliação de resultados (cuidados de saúde), esquizofrenia, serviços de saúde mental. escore médio global de 3,64. A média global das mudanças percebidas pelos pacientes foi de 2,46. Os principais preditores de melhor QV foram: em primeiro lugar, o escore global de mudança percebida e os escores das subescalas "Aspectos psicológicos e sono" e "Ocupação e saúde física". Outros três preditores foram: estar trabalhando, tomar a medicação sozinho e fazer uso de medicação apenas do tipo oral. Conclusão: A percepção de mudanças pelo próprio paciente, em função do tratamento, é um fator preditivo importante da QV.

\begin{abstract}
Objective: To investigate the factors associated to the quality of life (QOL) of patients with schizophrenia, specially their own perception of changes as a result of treatment received in mental health services. Method: The study was conducted in Divinópolis (MG), with patients attending the Reference Service for Mental Health. Individual structured interviews were conducted in order to apply the Quality of Life Scale (QLS-BR), the Scale of Perceived Change (EMP) and socio-demographic and clinical questionnaire. Three multiple linear regression analysis were performed in order to determine the relative contributions of differents predictors of QOL. Results: Participated in this study 72 patients, mostly males (59.7\%), diagnosed with paranoid schizophrenia (87.5\%). The patients' QOL fitted into the category of considerable damage, with a global average score of 3.64. The global average score of perceived chan-
\end{abstract}

1 Universidade Federal de São João del-Rei (UFSJ), Departamento de Psicologia, Laboratório de Pesquisa em Saúde Mental (LAPSAM).

Endereço para correspondência: Marina Bandeira e Luciana Cesari

Departamento de Psicologia, Laboratório de Pesquisa em Saúde Mental (LAPSAM), UFSJ

Praça Dom Helvécio, 74 - 36301-160 - São João del-Rei, MG

E-mails: bandeira@ufsj.edu.br e lucesari_psic@yahoo.com.br 


\section{Keywords}

Quality of life, outcome assessment (health care), schizophrenia, mental health services. ges was 2.46. The main predictors of better QOL were: firstly, the global score of perceived change and two subscales scores of "Psychological aspects and sleeping condition" and "Occupation and physical health". Other three predictors were: to be working, to take medication by oneself and to use only oral type medication. Conclusion: The patients perceived changes due to treatment outcome, is an important predictive factor of patients' QOL.

\section{INTRODUÇÃO}

O foco das pesquisas em saúde mental tem se voltado, cada vez mais, para a avaliação da qualidade dos serviços comunitários, que visam, prioritariamente, à reinserção social e à qualidade de vida (QV) dos pacientes com transtornos psiquiátricos ${ }^{1,2}$. A Organização Mundial da Saúde (OMS) tem apontado para a necessidade de avaliar continuamente esses serviços, com uma abordagem integrativa, que inclua as perspectivas dos profissionais dos serviços, familiares e pacientes ${ }^{3}$. De acordo com a OMS, os serviços de saúde mental devem proporcionar aos pacientes cuidados clínicos e não clínicos relevantes, visando reduzir o impacto da doença mental e melhorar sua QV나. Para isso, torna-se necessário avaliar a QV dos pacientes com transtornos psiquiátricos, em particular os portadores de transtornos mais graves, como a esquizofrenia 2,5 .

A esquizofrenia é uma doença crônica, com importante carga financeira e social para o paciente, seus familiares e a sociedade. Essa carga refere-se aos custos com o tratamento, à perda da produtividade e do status funcional, às limitações impostas pela doença e à diminuição da $\mathrm{QV}^{6}$. Um dos focos dos estudos nessa área tem sido o de investigar quais são os fatores que contribuem para uma melhor QV dos pacientes com esquizofrenia ${ }^{2}$.

No contexto da avaliação dos serviços de saúde mental, questiona-se em que grau os resultados do tratamento constituem um fator que contribui para uma melhor QV dos pacientes ${ }^{2,7-18}$. A avaliação dos resultados do tratamento tem sido considerada uma parte importante da avaliação dos serviços, em particular com medidas relatadas pelos próprios pacientes, ou Patient-Reported Outcomes, também denominados instrumentos $\mathrm{PRO}^{19}$. Dentre essas medidas, destacou-se, recentemente, a avaliação das mudanças percebidas pelos próprios pacientes, como resultado do tratamento recebido, na sua condição clínica, bem-estar físico, autonomia, bem-estar psicológico e na sua vida em geral ${ }^{20}$.

Em uma busca nas bases do Medline, SciELO e Lilacs, foram encontrados 12 estudos que procuraram identificar se os resultados obtidos com o tratamento psiquiátrico seriam um dos fatores determinantes que contribuem para uma melhor QV em pacientes com transtornos psiquiátricos, principalmente entre pacientes com diagnóstico de esquizofrenia $^{7-18}$. De forma geral, esses estudos mostraram que a QV desses pacientes depende da ocorrência de resultados positivos do tratamento recebido nos serviços de saúde mental, em termos da diminuição da sintomatologia, meIhor condição mental e maior grau de funcionalidade dos pacientes, geralmente avaliados por profissionais. Dentre esses estudos, apenas dois utilizaram medidas de resultados relatados pelos próprios pacientes ${ }^{8,12}$ e nenhum deles incluiu a aferição da percepção de mudanças. Há, portanto, carência de estudos sobre essa temática. O objetivo do presente trabalho foi investigar em que grau a percepção de mudanças pelos próprios pacientes, como resultado do tratamento, seria um fator preditivo da $\mathrm{QV}$ de pacientes com esquizofrenia, comparando-se também diferentes variáveis sociodemográficas e clínicas.

\section{MÉTODO}

\section{Delineamento}

Este estudo consiste em uma pesquisa avaliativa, do tipo somativa ou dos efeitos, que foi definida por Selltiz et al. ${ }^{21}$ como uma pesquisa que avalia os resultados de um serviço, programa de intervenção ou tratamento, contrariamente a uma pesquisa que avalia o processo, esta última denominada pesquisa avaliativa do tipo formativa. O desenho do estudo é de corte transversal, tendo em vista que as medidas foram tomadas em um mesmo momento no tempo ${ }^{22}$. O estudo visa avaliar relações entre variáveis, sem manipulação experimental, ou seja, sem a introdução de uma variável de tratamento aos pacientes.

\section{Participantes}

Participaram deste estudo pacientes que residiam na cidade de Divinópolis (MG) e que recebiam tratamento ambulatorial no Serviço de Referência em Saúde Mental (Sersam) da referida cidade. Segundo dados do IBGE, Divinópolis está localizada na mesorregião geográfica oeste de Minas Gerais e a estimativa do número de habitantes é de 216 mil. Além do Sersam, a cidade conta com um hospital psiquiátrico. Os pacientes foram incluídos na amostra conforme os seguintes critérios: diagnóstico de esquizofrenia indicado no prontuário, classificado segundo a Classificação Internacional de Doenças da OMS (CID-10)23; evolução mínima da doença de um ano; tempo de tratamento no serviço superior a um ano; ter idade entre 18 e 55 anos; ausência de alteração da medicação nos últimos três meses e quadro clínico estável nas três 
semanas precedentes à coleta de dados. Foram adotados os seguintes critérios de exclusão: ter diagnóstico de doença neurológica, comprometimento cognitivo ou dependência de drogas ilícitas e álcool, apresentar dificuldade em compreender as questões das escalas e instruções da entrevista.

A amostra foi do tipo não probabilística e constituiu-se de toda a população-alvo elegível do serviço que estava acessível e que atendeu à solicitação de participação na pesquisa. O total de sujeitos elegíveis no serviço, que preenchiam os critérios de inclusão e exclusão adotados, foi de 156. Todos esses pacientes elegíveis foram convidados a participar do estudo por meio de telefonemas e cartas, cujo remetente era o próprio serviço. Para os contatos realizados por meio das cartas, foram conduzidas três tentativas. Do total de 81 contatos feitos por cartas, obteve-se a participação de 20 pacientes. De 75 contatos feitos por telefone, obteve-se a participação de 52 pacientes. No total, participaram deste estudo 72 pacientes.

\section{Instrumentos de medida}

Foram utilizados três instrumentos de medida: Escala de Qualidade de Vida (QLS-BR), Escala de Mudança Percebida (EMP) e Questionário Sociodemográfico e Clínico.

Escala QLS-BR: Esta escala possui 21 itens que avaliam a QV de pacientes com diagnóstico de esquizofrenia, por meio de uma entrevista semiestruturada. As alternativas de resposta para os itens estão dispostas em uma escala do tipo Likert de sete pontos, cuja marcação fica sob julgamento do entrevistador, após questionar os pacientes a respeito de cada item. Os escores 5 e 6 indicam funcionamento inalterado; 2 a 4, considerável prejuízo e 0 ou 1 refletem prejuízo grave. A escala abrange três domínios da vida dos pacientes: rede social, nível ocupacional, funções intrapsíquicas e relações interpessoais. O estudo das qualidades psicométricas dessa escala mostrou indicadores de validade e fidedignidade adequados ${ }^{24}$.

Escala EMP: Esta escala possui 19 itens que avaliam as mudanças percebidas pelos próprios pacientes, em função do tratamento recebido. Essa avaliação independe do diagnóstico do paciente e é realizada por meio da aplicação da escala, em situação de entrevista estruturada. Dentre os itens da escala, 18 avaliam as mudanças percebidas pelo paciente em três dimensões de suas vidas: ocupação e saúde física, dimensão psicológica e sono, relacionamentos e estabilidade emocional. O último item (item 19) avalia, globalmente, as mudanças percebidas na vida, em geral. As alternativas de resposta aos itens estão dispostas em uma escala do tipo $\mathrm{Li}$ kert de três pontos, sendo 1 pior do que antes, 2 sem mudança e 3 melhor do que antes. As perguntas e as alternativas de resposta são lidas pelo entrevistador, que marca a resposta indicada oralmente pelo paciente. Essa escala foi elaborada por Mercier et al..20, tendo sido adaptada para o Brasil por Bandeira et al..$^{25}$ e validada por Bandeira et al. ${ }^{26}$. O estudo das qualidades psicométricas dessa escala também mostrou indicadores de validade e fidedignidade adequados ${ }^{26}$.

Questionário sociodemográfico e clínico: Para a caracterização da amostra de pacientes, foi utilizado um questionário sociodemográfico e clínico que incluiu 44 questões, elaborado especificamente para este estudo. Este questionário foi submetido a uma comissão de especialistas e a um estudo piloto, visando avaliar sua adequação aos objetivos da pesquisa e a clareza das questões. Para as questões dicotômicas, optou-se pela seguinte categorização das respostas: $1 \mathrm{Sim}$; 2 Não.

\section{Procedimento}

Para a realização deste estudo, foi obtido, primeiramente, o consentimento da direção do Sersam e da Secretaria Municipal de Saúde da cidade de Divinópolis. Em seguida, o projeto foi encaminhado à Comissão de Ética em Pesquisa com Seres Humanos da Universidade Federal de São João del-Rei (UFSJ), sendo aprovado conforme o memorando 007/2008.

A coleta de dados foi realizada entre os meses de janeiro e junho de 2009. Foram feitas entrevistas individuais com os pacientes, nas dependências do SERSAM, por uma psicóloga previamente treinada para esse fim. Foi solicitada, aos pacientes, a assinatura de um Termo de Consentimento Livre e Esclarecido. As entrevistas foram realizadas conforme a seguinte ordem de aplicação dos instrumentos: QLS-BR, EMP e questionário sociodemográfico e clínico. Não houve conflito de interesse nesta investigação.

\section{Análise de dados}

Os dados coletados foram computados no programa Statistical Package for the Social Sciences (SPSS-PC), versão 13.0. Foram realizadas análises estatísticas descritivas dos dados, com o cálculo das médias e porcentagens das variáveis sociodemográficas e clínicas, assim como dos escores das escalas EMP e QLS-BR. Para avaliar as diferenças entre os escores médios das subescalas da EMP e QLS-BR, foram realizadas análises de variância (ANOVA) e teste post hoc de Tukey. Essas análises foram realizadas após a verificação da normalidade dos dados globais de percepção de mudança e QV, pelo teste Kolmogorov-Smirnov.

Para a análise dos fatores associados à QV, foi considerada como variável dependente, também denominada variável resposta, a média global da QLS-BR. Primeiramente, foram realizadas análises estatísticas univariadas, visando identificar as variáveis a serem incluídas na análise de regressão posterior. Para isso, utilizou-se o teste $t$ de Student para amostras independentes, para a comparação de grupos e a análise de correlação de Pearson, para variáveis contínuas. As variáveis sociodemográficas, clínicas e de percepção de mudança que apresentaram relação significativa com a QV $(p \leq 0,05)$ foram selecionadas para as análises multivariadas. Conforme a indicação da literatura da área sobre a criação de modelos 
de regressão linear ${ }^{27}$, incluiu-se também, nessas análises, as variáveis não significativas com valor de " $p$ " de até 0,25.

Após a verificação da normalidade das variáveis referentes aos escores das escalas EMP e QLS, por meio dos testes Kolmogorov-Smirnov e Durbin-Watson e da homocedasticidade dos dados e independência dos resíduos, pela análise dos gráficos P-P, foram conduzidas três análises de regressão linear múltipla para identificar os fatores associados à QV. Os modelos de regressão foram construídos por meio da técnica stepwise $e^{28}$ e incluíram a constante, já que era possível atribuir a pontuação 0 às questões da escala QLS-BR ${ }^{29}$. Foram feitas três análises de regressão. A primeira análise foi realizada apenas com as variáveis sociodemográficas e clínicas, visando identificar inicialmente, dentro dessa categoria de variáveis, quais delas contribuíram mais para uma melhor QV, sem a interferência de outras categorias de variáveis. A segunda análise de regressão incluiu as variáveis sociodemográficas e clínicas e os escores médios das três subescalas da EMP, visando identificar se as subescalas de percepção de mudanças teriam uma maior contribuição para a QV, comparativamente às variáveis sociodemográficas e clínicas, e quais dessas subescalas se destacariam nessa relação. A terceira análise de regressão incluiu, além das variáveis sociodemográficas e clínicas, o escore global médio da EMP, visando identificar se a percepção global de mudança teria maior contribuição na QV dos que as demais variáveis. As medidas de escore global e das subescalas de percepção de mudança foram separadas em dois modelos distintos de regressão, para evitar a colinearidade entre elas, tendo em vista que o escore global consiste na média das subescalas. As análises feitas, por meio desses três modelos de regressão, permitiram, portanto, verificar o efeito isolado e combinado das diversas variáveis na determinação da QV. O critério para permanência de uma variável nos modelos finais de regressão foi o valor de $p \leq 0,05$. A ordenação das variáveis, como fatores preditores da $\mathrm{QV}$, seguiu a ordem dos valores do coeficiente Beta, obtidos na análise de regressão.

\section{RESULTADOS}

\section{Descrição da amostra}

Os resultados referentes às características sociodemográficas e clínicas dos pacientes estão dispostos na tabela 1. A média de idade encontrada foi de 40,6 anos. Dentre os pacientes, $59,7 \%$ eram do sexo masculino. A maioria $(58,3 \%)$ possuía o primeiro grau incompleto. Com relação ao estado conjugal, a maioria vivia sem um companheiro (83,3\%). Quanto à renda, a maior parte dos pacientes $(69,4 \%)$ possuía alguma fonte de renda e, dentre esses, $92 \%$ recebiam menos de dois salários-mínimos. Quanto à situação de trabalho, a maioria (79,2\%) não trabalhava e 51,4\% eram aposentados.
Tabela 1. Frequências absolutas (n) e relativas (\%) das variáveis sociodemográficas e clínicas dos pacientes

\begin{tabular}{|c|c|c|}
\hline Variáveis & $\mathrm{n}$ & $\%$ \\
\hline \multicolumn{3}{|l|}{ Sexo } \\
\hline Masculino & 43 & 59,7 \\
\hline Feminino & 29 & 40,3 \\
\hline \multicolumn{3}{|l|}{ Escolaridade } \\
\hline Analfabeto e escola especial & 3 & 4,2 \\
\hline Ensino fundamental incompleto & 42 & 58,3 \\
\hline Ensino fundamental completo ou mais & 27 & 37,5 \\
\hline \multicolumn{3}{|l|}{ Situação conjugal } \\
\hline Vivendo com companheiro & 12 & 16,7 \\
\hline Vivendo sem companheiro & 60 & 83,3 \\
\hline \multicolumn{3}{|l|}{ Recebe renda } \\
\hline Sim & 50 & 69,4 \\
\hline Não & 22 & 30,6 \\
\hline \multicolumn{3}{|l|}{ Valor da renda } \\
\hline$\leq 2$ salários & 46 & 92 \\
\hline > 2 salários & 3 & 6 \\
\hline Não soube informar & 1 & 2 \\
\hline \multicolumn{3}{|l|}{ Se o paciente é aposentado } \\
\hline $\operatorname{Sim}$ & 37 & 51,4 \\
\hline Não & 35 & 48,6 \\
\hline \multicolumn{3}{|l|}{ Se o paciente trabalha } \\
\hline $\operatorname{Sim}$ & 15 & 20,8 \\
\hline Não & 57 & 79,2 \\
\hline \multicolumn{3}{|l|}{ Diagnóstico (CID10) } \\
\hline Esquizofrenia paranoide & 63 & 87,4 \\
\hline Outras* & 9 & 12,6 \\
\hline \multicolumn{3}{|l|}{ Realiza outros tratamentos médicos } \\
\hline Sim & 31 & 43 \\
\hline Não & 41 & 57 \\
\hline \multicolumn{3}{|l|}{ Atividades realizadas no serviço de saúde mental } \\
\hline Apenas consulta & 22 & 30,6 \\
\hline Consulta e pegar a medicação & 45 & 62,5 \\
\hline $\begin{array}{l}\text { Consulta, pegar a medicação e participar de } \\
\text { atividades terapêuticas }\end{array}$ & 5 & 6,9 \\
\hline \multicolumn{3}{|l|}{ Frequência de comparecimento no serviço } \\
\hline Consulta e outros & 50 & 69,4 \\
\hline Apenas consulta & 22 & 30,6 \\
\hline \multicolumn{3}{|l|}{ Forma de administração da medicação } \\
\hline Oral & 60 & 83,3 \\
\hline Injetável & 1 & 1,4 \\
\hline Oral e injetável & 11 & 15,3 \\
\hline \multicolumn{3}{|l|}{ Autonomia do paciente no uso da medicacão } \\
\hline Sim & 48 & 66,7 \\
\hline Não & 24 & 33,3 \\
\hline
\end{tabular}

* Nesta categoria, estão incluídos: esquizofrenia hebefrênica, esquizofrenia indiferenciada, esquizofrenia não especificada e sem a descrição do subtipo.

A maioria dos pacientes $(87,4 \%)$ possuía o diagnóstico de esquizofrenia paranoide. A duração média da doença foi de 16,3 anos e a duração média do tratamento no serviço foi de 8,6 anos. Dos entrevistados, $43 \%$ recebiam outros tratamentos médicos. Os pacientes tomavam, em média, 3,1 medicamentos, e a maioria (83,3\%) tomava apenas medicação oral. A média de tempo de inalteração da medicação foi de 22,3 meses. A maioria dos pacientes estudados $(93,1 \%)$ recebia apenas tratamento ambulatorial, não participando de nenhuma atividade terapêutica no serviço. 


\section{Qualidade de vida}

Os escores obtidos na escala QLS-BR foram submetidos ao teste Kolmogorov-Smirnov, apresentando valor de p igual a 0,94 (escore $\mathrm{K}-\mathrm{S}=0,53$ ), o que indica uma distribuição normal desses dados. Os valores da média global e dos três domínios de QV se enquadraram na categoria de considerável prejuízo no funcionamento dos pacientes (Tabela 2). A média global de QV foi de 3,64 e, para as subescalas, os valores variaram entre 3,53, no domínio "Rede social", e 4,01, no domínio "Nível ocupacional". Os escores médios dos três domínios foram submetidos a uma análise comparativa, por meio da análise de variância (ANOVA) e teste post hoc de Tukey. Os resultados mostraram que o grau de QV não diferiu nas três dimensões avaliadas.

Com relação à categorização da QV, por grau de prejuízo na vida do paciente (Tabela 2), pode-se constatar que as maiores porcentagens de pacientes se enquadraram na categoria de considerável prejuízo, para todas as dimensões avaliadas, com destaque para a dimensão "Funções intrapsíquicas e relações interpessoais" (84,7\%) e a dimensão "Rede social" (73,6\%). Pode-se observar, igualmente, que a dimensão "Nível ocupacional" apresentou a maior porcentagem de pacientes com funcionamento inalterado (37,5\%), comparativamente às outras dimensões. A categoria de "Prejuízo grave" incluiu uma baixa porcentagem de pacientes.

\section{Mudanças percebidas pelos pacientes}

Os escores globais obtidos na escala EMP foram submetidos ao teste Kolmogorov-Smirnov, que apresentou valor de p igual a 0,13 (escore $K-S=1,16$ ), indicando uma distribuição normal desses dados. A tabela 3 apresenta os escores médios de percepção de mudança para a escala global e para as três subescalas da EMP. Encontrou-se um escore médio global igual a 2,46 . Os resultados das subescalas mostraram que as médias obtidas variaram de 2,31, na dimensão "Ocupação e saúde física", a 2,62, na dimensão "Aspectos psicológicos e sono".

Para comparação dos dados das três subescalas, foi feita uma análise de variância (ANOVA) e teste post hoc de Tukey e os resultados mostraram que o grau de melhora percebida pelos pacientes foi significativamente mais elevado na di- mensão "Aspectos psicológicos e sono" (subescala 2), comparativamente às demais dimensões. Essa subescala avalia os seguintes aspectos da vida dos pacientes: autoconfiança, humor, problemas pessoais, interesse pela vida, enfrentamento de situações difíceis e qualidade do sono. As médias das outras duas subescalas não apresentaram diferenças estatisticamente significativas entre si.

Pode-se observar ainda, na tabela 3, as porcentagens de pacientes que perceberam melhora, piora ou ausência de mudanças, em cada item da escala EMP. Os resultados mostraram que a maioria dos pacientes percebeu uma melhora, em geral, em suas vidas $(88,9 \%)$ em função do tratamento recebido (item 19, global). Resultados mais diferenciados foram obtidos para os 18 itens específicos das subescalas. $\mathrm{O}$ item que apresentou maior porcentagem de melhora percebida $(81,9 \%)$ foi o que se refere aos problemas pessoais dos pacientes. O item com maior índice de ausência de mudança se referiu à sexualidade, para o qual 62,5\% dos pacientes relataram não ter percebido nenhuma mudança, em função do tratamento. $\mathrm{O}$ item com maior índice de piora foi o da saúde física, no qual $26,4 \%$ dos pacientes relataram ter percebido uma piora, em função do tratamento, seguido dos itens energia $(23,6 \%)$ e sexualidade $(20,8 \%)$.

\section{Análise univariada}

A descrição completa dessas análises encontra-se em Cesa$\mathrm{ri}^{30}$, sendo apresentada resumidamente a seguir. Os resultados das análises univariadas dos fatores associados à QV global (Tabela 4) mostraram que os pacientes que tomavam a medicação sozinhos $(p=0,01)$, que trabalhavam $(p=0,001)$, viviam com um companheiro $(p=0,02)$ e que tomavam apenas medicação do tipo oral $(p=0,03)$ apresentaram QV significativamente melhor, quando comparados aos pacientes que precisavam de ajuda para tomar seus medicamentos, não trabalhavam, não viviam com um companheiro e que faziam uso conjunto de medicação psiquiátrica oral e injetável. Os dados da medicação utilizados na presente pesquisa não incluíam a descrição dos tipos específicos de medicamento, apenas a forma de administração. Além disso, quanto maior foi o número de meses de uso da mesma medicação, melhor foi a QV dos pacientes $(p=0,03)$.

Tabela 2. Médias dos escores de qualidade de vida para a escala global e subescalas da QLS-BR e porcentagens e frequências absolutas (n) dos pacientes classificados nas categorias de funcionamento por subescala

\begin{tabular}{|c|c|c|c|c|c|c|c|}
\hline \multirow[t]{2}{*}{ Subescalas e escala global } & \multicolumn{2}{|c|}{$\begin{array}{l}\text { Prejuízo grave } \\
(0-1,99)\end{array}$} & \multicolumn{2}{|c|}{$\begin{array}{c}\text { Considerável prejuízo } \\
(2-4,99)\end{array}$} & \multicolumn{2}{|c|}{$\begin{array}{l}\text { Funcionamento inalterado } \\
\qquad(5 \mathrm{a} 6)\end{array}$} & \multirow[t]{2}{*}{ Médias } \\
\hline & n & $\%$ & n & $\%$ & n & $\%$ & \\
\hline Rede social & 6 & 8,3 & 53 & 73,6 & 13 & 18,1 & 3,53 \\
\hline Nível ocupacional & 6 & 8,3 & 39 & 54,2 & 27 & 37,5 & 4,01 \\
\hline Funções intrapsíquicas e relações interpessoais & 5 & 6,9 & 61 & 84,7 & 6 & 8,3 & 3,58 \\
\hline Escore global & 4 & 5,6 & 60 & 83,3 & 8 & 11,1 & 3,64 \\
\hline
\end{tabular}

$\mathrm{F}=3,17 ; \mathrm{p}=0,06$. 
Tabela 3. Médias dos escores de percepção de mudança pelos pacientes, para a escala global e subescalas da EMP e porcentagens de pacientes que perceberam melhora, ausência de mudança ou piora, em cada item da escala

\begin{tabular}{|c|c|c|c|c|c|}
\hline Subescalas, item global e escala global & Itens & \% Piora & \% Sem mudança & \% Melhora & Médias \\
\hline \multirow[t]{8}{*}{ Ocupação e saúde física } & Atividades de lazer & 16,7 & 31,9 & 51,4 & 2,31 \\
\hline & Energia & 23,6 & 23,6 & 52,8 & \\
\hline & Tarefas de casa & 4,2 & 45,8 & 50 & \\
\hline & Capacidade de cumprir as obrigações e tomar decisões & 5,6 & 34,7 & 59,7 & \\
\hline & Interesse em trabalhar ou se ocupar com alguma coisa & 13,9 & 29,2 & 56,9 & \\
\hline & Sexualidade & 20,8 & 62,5 & 16,7 & \\
\hline & Apetite & 13,9 & 33,3 & 52,8 & \\
\hline & Saúde física & 26,4 & 44,4 & 29,2 & \\
\hline \multirow[t]{6}{*}{ Aspectos psicológicos e sono } & Sentimento de confiança em si próprio & 6,9 & 19,5 & 73,6 & $2,62^{*}$ \\
\hline & Humor & 5,6 & 33,3 & 61,1 & \\
\hline & Problemas pessoais & 4,2 & 13,9 & 81,9 & \\
\hline & Sentimento de interesse pela vida & 4,2 & 40,2 & 55,6 & \\
\hline & Capacidade de suportar situações difíceis & 5,6 & 37,5 & 56,9 & \\
\hline & Sono & 9,7 & 12,5 & 77,8 & \\
\hline \multirow[t]{4}{*}{ Relacionamentos e estabilidade emocional } & Convivência com amigos e amigas & 9,7 & 59,7 & 30,6 & 2,44 \\
\hline & Estabilidade das emoções & 8,4 & 45,8 & 45,8 & \\
\hline & Convivência com outras pessoas & 5,6 & 33,3 & 61,1 & \\
\hline & Convivência com a família & 6,9 & 25 & 68,1 & \\
\hline Item global & Avaliação global das mudanças & 1,4 & 9,7 & 88,9 & \\
\hline Escala global & & _ & & & 2,46 \\
\hline
\end{tabular}

Tabela 4. Variáveis selecionadas para as análises multivariadas

\begin{tabular}{|c|c|c|c|c|}
\hline Categorias & Variáveis significativas com $p \leq 0,05$ & $\begin{array}{l}\text { Valores das análises } \\
\text { univariadas }\end{array}$ & Variáveis com $p \leq 0,25$ & $\begin{array}{l}\text { Valores das análises } \\
\text { univariadas }\end{array}$ \\
\hline \multirow[t]{5}{*}{ Variáveis sociodemográficas e clínicas } & Se o paciente toma o medicamento sozinho & $t=2,55$ & Se o paciente faz outros tratamentos médicos & $t=1,55$ \\
\hline & Se o paciente trabalha & $t=3,60$ & Frequência de comparecimento no serviço & $t=1,25$ \\
\hline & Se o paciente vive com companheiro & $t=-2,43$ & Se o paciente tem renda & $t=1,65$ \\
\hline & Tipo de medicação & $t=2,23$ & & \\
\hline & Número de meses de inalteração da medicação & $r=0,25$ & & \\
\hline \multirow[t]{4}{*}{ Escores de percepção de mudança } & EMP global & $r=0,76$ & & \\
\hline & EMP ocupação e saúde física & $r=0,64$ & & \\
\hline & EMP aspectos psicológicos e sono & $r=0,66$ & & \\
\hline & EMP relacionamentos e estabilidade emocional & $r=0,64$ & & \\
\hline
\end{tabular}

r: correlação de Pearson; t: teste t de Student.

Para as variáveis de percepção de mudança, todas as análises realizadas apresentaram resultados significativos ( $p$ $<0,01)$. Os dados obtidos mostraram que quanto maior era o escore global de mudança percebida (média dos 18 itens da escala) e os escores de percepção de mudanças nas três subescalas da EMP, melhor era a QV dos pacientes.

Outras três variáveis apresentaram relação com a QV, com valores de $p \leq 0,25$. Foram elas: se o paciente realiza outros tratamentos médicos $(p=0,13)$, a frequência de comparecimento no serviço $(p=0,22)$ e se o paciente possui fonte de renda $(p=0,10)$.

\section{Análise de regressão linear múltipla}

Os resultados referentes às análises de regressão encontram-se dispostos na tabela 5, que apresenta também o valor do índice de Durbin-Watson (DW), que corrobora a hipótese de distribuição normal dos dados. Na primeira análise, que incluiu apenas as variáveis sociodemográficas e clínicas, os resultados mostraram que a QV foi melhor para os pacientes que tomavam suas medicações sozinhos, faziam uso de medicação do tipo oral e trabalhavam. O valor do $R^{2}$ ajustado mostrou que essas três variáveis explicaram $28 \%$ da contribuição das variáveis sociodemográficas e clínicas para o grau de QV. 
Tabela 5. Resultados das análises de regressão linear para os fatores associados à qualidade de vida

\begin{tabular}{|c|c|c|c|c|c|c|}
\hline Modelos & Variável preditora & Beta & Erro-padrão & $\mathrm{t}$ & p & \\
\hline \multirow[t]{4}{*}{ Variáveis sociodemográficas e clínicas } & Constante & $+7,19$ & 0,65 & 11,07 & 0,00 & \multirow{4}{*}{$\begin{array}{l}R^{2}=0,28 \\
F(4,03)=10,29 \\
(p=0,00) \\
D W=1,83\end{array}$} \\
\hline & Se o paciente trabalha & $-1,15$ & 0,27 & $-4,19$ & 0,00 & \\
\hline & Se o paciente toma o medicamento sozinho & $-0,56$ & 0,24 & $-2,37$ & 0,02 & \\
\hline & Tipo de medicação & $-0,62$ & 0,31 & $-2,01$ & 0,04 & \\
\hline \multirow{5}{*}{$\begin{array}{l}\text { Variáveis sociodemográficas e clínicas e os } \\
\text { escores das subescalas de percepção de mudança }\end{array}$} & Constante & $+0,09$ & 0,85 & 0,11 & 0,91 & \multirow{2}{*}{$\begin{array}{l}R^{2}=0,65 \\
F(10,30)=33,16 \\
(p=0,00)\end{array}$} \\
\hline & EMP aspectos psicológicos e sono & $+1,19$ & 0,26 & 4,65 & 0,00 & \\
\hline & EMP ocupação e saúde física & $+1,03$ & 0,24 & 4,31 & 0,00 & $\mathrm{DW}=1,53$ \\
\hline & Se o paciente trabalha & $-0,69$ & 0,20 & $-3,46$ & 0,001 & \\
\hline & Se o paciente toma o medicamento sozinho & $-0,53$ & 0,16 & $-3,20$ & 0,002 & \\
\hline \multirow{4}{*}{$\begin{array}{l}\text { Variáveis sociodemográficas e clínicas e o escore } \\
\text { global de percepção de mudança }\end{array}$} & Constante & $-0,58$ & 0,89 & $-0,65$ & 0,52 & \multirow{4}{*}{$\begin{array}{l}R^{2}=0,66 \\
F(8,88)=45,94 \\
(p=0,00) \\
D W=1,42\end{array}$} \\
\hline & EMP global & $+2,44$ & 0,27 & 9,04 & 0,00 & \\
\hline & Se o paciente toma o medicamento sozinho & $-0,52$ & 0,16 & $-3,24$ & 0,002 & \\
\hline & Se o paciente trabalha & $-0,60$ & 0,20 & $-2,98$ & 0,004 & \\
\hline
\end{tabular}

A segunda análise de regressão incluiu, como variáveis independentes, os escores das subescalas de percepção de mudança, além das variáveis sociodemográficas e clínicas dos pacientes. Os resultados destacaram os escores de percepção de mudança na predição da QV. Os fatores que foram mais importantes para predizer uma melhor QV foram: em primeiro lugar, apresentar maior percepção de melhora na subescala "Aspectos psicológicos e sono" e maior percepção de melhora na dimensão "Ocupação e saúde física", em seguida tomar a medicação sozinho e estar trabalhando. Essas quatro variáveis explicaram $65 \%$ da contribuição de todas as variáveis para o grau de QV.

Na terceira análise de regressão, que incluiu o escore global de percepção de mudança, além das variáveis sociodemográficas e clínicas, os resultados apontaram que os principais fatores associados a uma melhor QV foram: em primeiro lugar, apresentar maior grau de percepção global de melhora, em seguida tomar a medicação sozinho e estar trabalhando. Essas três variáveis explicaram $66 \%$ da contribuição de todas as variáveis para o grau de QV.

\section{DISCUSSÃO}

A partir dos resultados encontrados, constatou-se que a QV dos pacientes com esquizofrenia foi determinada, principalmente, pelo grau de melhora percebida pelos próprios pacientes em suas vidas, em função do tratamento recebido no serviço de saúde mental, avaliado pela escala EMP. Outros 12 estudos encontraram resultados semelhantes, constatando que a QV estava relacionada com os resultados do tratamento recebido, embora utilizando medidas diferentes de resultados, que não a percepção de mudanças ${ }^{7-18}$. Esses estudos avaliaram amostras que eram compostas, principal- mente, por pacientes com esquizofrenia e transtorno esquizoafetivo, com exceção da pesquisa de Russo et al. ${ }^{8}$, que foi constituída por pacientes com diferentes diagnósticos, entre eles depressão (unipolar e bipolar), esquizofrenia, transtorno esquizoafetivo, mania aguda e outros diagnósticos.

Desses 12 estudos, 10 avaliaram os resultados do tratamento com medidas tomadas por clínicos treinados, e não por avaliações de resultados relatados pelos próprios pacientes. As avaliações dos clínicos envolveram, em geral, medidas da sintomatologia, do estado de saúde mental e do nível de funcionamento dos pacientes. Com relação à gravidade da sintomatologia, constatou-se, em sete estudos, que uma menor gravidade dos sintomas, mensurada pela Scale for the Assessment of Negative Symptoms (SANS) ou Positive and Negative Syndrome Scale (PANSS), associou-se a uma melhor $\mathrm{QV}$, avaliada por diferentes escalas $7,9,1,13,15,17,18$. No que se refere ao estado de saúde mental dos pacientes, encontrou-se, em dois estudos, que um melhor estado de saúde mental, avaliado pela escala Brief Psychiatric Rating Scale (BPRS), se relacionou com uma melhor $\mathrm{QV}$, mensurada por diferentes instrumentos ${ }^{14,16}$. Quanto ao nível de funcionamento, os resultados de quatro estudos indicaram que quanto melhor era o funcionamento global dos pacientes, avaliado pelas escalas Global Assessment of Functioning Scale (GAF) ou Clinical Global Impression (CGI), melhor era a QV, mensurada por diferentes escalas ${ }^{9-11,14}$.

Apenas dois estudos, dentre os 12 citados, avaliaram a relação entre QV e medidas de resultados relatados pelos próprios pacientes, como o presente trabalho ${ }^{8,12}$. Porém, essas medidas adotadas nos dois estudos avaliavam o estado de saúde mental e a sintomatologia dos pacientes, diferentemente do presente trabalho. Por exemplo, Russo et al. ${ }^{8}$ encontraram que quanto menor era a gravidade dos sintomas, mensurada pela Behavior and Symptom Identification Scale 
(BASIS-32), melhor era a QV dos pacientes, avaliada pela escala Lehman's Quality of Life Interview (QOLI). Wilkinson et al. ${ }^{12}$ encontraram que quanto melhor era o estado de saúde mental dos pacientes, avaliado pela escala de Goldberg General Health Questionnaire (GHQ-12), melhor era a QV, mensurada pela escala Schizophrenia Quality of life Scale (SQLS). Sendo assim, a presente pesquisa constatou um resultado inédito em relação aos 12 estudos encontrados, tendo indicado que a percepção de mudança, tal como relatada pelos próprios pacientes, constituiu a variável preditiva mais importante de melhor QV.

A avaliação da QV, na presente pesquisa, permitiu constatar que os pacientes se enquadraram na categoria de considerável prejuízo no funcionamento, nos dados tanto do escore global quanto dos escores médios dos três domínios da escala. Outros dois estudos realizados com a escala QLS-BR encontraram resultados semelhantes. Cardoso et al.24 constataram considerável prejuízo para os dados do escore global de QV e para as três dimensões da escala QLS-BR, enquanto Santana et al. ${ }^{31}$ observaram considerável prejuízo apenas para a dimensão "Funções intrapsíquicas e relações interpessoais" dessa escala.

Com relação aos fatores associados à QV, a primeira análise de regressão linear múltipla, que se restringiu às variáveis sociodemográficas e clínicas, indicou que os pacientes que tomavam a medicação sozinhos faziam uso apenas de medicação oral e apresentaram melhor QV. Outros estudos também encontraram que a variável "trabalho" estava associada a uma melhor $\mathrm{QV}^{14,18,32,33}$. Com relação às outras duas variáveis, uma possível explicação para o resultado encontrado é que os pacientes que têm autonomia para tomar sua medicação e que fazem uso apenas de medicação oral provavelmente apresentam um quadro clínico menos grave e aderem meIhor ao tratamento, explicando o resultado de QV.

No segundo modelo de análise de regressão, que incluiu os escores das subescalas de percepção de mudanças, os resultados indicaram que os principais fatores preditivos de uma melhor QV dos pacientes foram as percepções de meIhora pelos pacientes em dois domínios: "Aspectos psicológicos e sono" e "Ocupação e saúde física", além das variáveis tomar a medicação sozinho e trabalhar. No terceiro modelo de análise de regressão, os resultados mostraram que a percepção de mudança global, bem como a autonomia no uso da medicação e o trabalho, foram os três fatores preditivos de uma melhor QV.

Destaca-se, portanto, no presente estudo, a capacidade de predição da medida de percepção de mudanças pelos próprios pacientes, como indicadora de melhor QV. Além da percepção de mudança, as variáveis "trabalho" e "autonomia no uso da medicação" também se mostraram importantes, podendo indicar que um maior nível de independência dos pacientes contribui para uma melhor QV.

Os resultados mostraram que o grau de percepção de melhora pelos pacientes foi maior na dimensão "Aspectos psicológicos e sono". Foram constatadas, também, maior percepção de melhora no item específico dos problemas pessoais dos pacientes, maior percepção de piora nas questões referentes à saúde física, energia e sexualidade e maior percepção de ausência de mudança no item da sexualidade. Costa $^{34}$, que também utilizou a escala EMP, encontrou resultados semelhantes. A autora também constatou maiores porcentagens de percepção de melhora na dimensão "Aspectos psicológicos e sono" e, em particular, no item referente aos problemas pessoais. Observou também que as questões referentes à sexualidade e à saúde física apresentaram elevadas taxas de ausência de mudança e piora, respectivamente. Ausência de mudança e piora referentes à sexualidade também foram observadas por Perreault et al. ${ }^{35}$, utilizando uma versão canadense da mesma escala de medida.

A presente pesquisa apresenta algumas limitações. Por se tratar de um estudo transversal e correlacional, é necessária cautela na interpretação dos resultados, visto que a análise correlacional não permite o estabelecimento de uma relação causal entre os construtos avaliados. Estudos futuros longitudinais poderiam fornecer uma informação mais precisa dessa relação ${ }^{26}$. Outra limitação é que parte dos pacientes elegíveis não compareceu para a realização das entrevistas, o que pode comprometer a representatividade da amostra. Para minimizar esse problema, foi analisada estatisticamente a equivalência entre os sujeitos que compareceram e os sujeitos faltosos, quanto a algumas variáveis sociodemográficas e clínicas. Constatou-se que os pacientes que participaram e aqueles que não participaram eram equivalentes quanto a sexo, idade, tempo de tratamento no serviço e número de remédios prescritos, embora tenham diferido quanto ao diagnóstico e tempo de tratamento psiquiátrico. Com relação ao diagnóstico, os pacientes que participaram eram, em sua maioria, portadores de esquizofrenia paranoide, o que pode limitar a generalidade dos dados para os demais subtipos de esquizofrenia. Quanto ao tempo de tratamento psiquiátrico, constatou-se que os pacientes que participaram realizavam tratamento psiquiátrico há mais tempo do que os que não participaram. Essas duas diferenças podem limitar a generalidade dos resultados para esse tipo de amostra. Os diagnósticos dos pacientes, identificados nos prontuários, não foram submetidos a uma reavaliação por especialistas, mas um estudo precedente, com uma amostra de pacientes com esquizofrenia, havia confirmado, em 95\% dos casos, a confiabilidade dos diagnósticos nesse mesmo serviço de saúde mental24.

\section{CONCLUSÕES}

O principal resultado deste estudo é que o fator preditivo mais importante da QV foi a percepção de mudança pelos próprios pacientes, em função do tratamento. Esses resultados confirmam a relevância da avaliação feita pelos próprios pacientes, 
na aferição dos resultados do tratamento, tendência que tem sido destacada na literatura da área, nos últimos anos.

Se a QV dos pacientes é um resultado a ser almejado pelos serviços de saúde mental, a avaliação da percepção de mudanças pode fornecer pistas importantes para atingir esse objetivo. Essa avaliação pode apontar os aspectos que necessitam ser melhorados no tratamento, quando o paciente percebe piora ou ausência de mudanças, em alguns aspectos de sua vida.

Portanto, as informações levantadas com a realização deste estudo são relevantes para os profissionais da área da saúde mental. Elas podem contribuir para o redimensionamento do tratamento, de forma a ajustá-lo às necessidades dos pacientes. Os serviços de saúde mental teriam a ganhar adotando, rotineiramente, avaliações contínuas dos resultados do tratamento, em particular, da percepção de mudança pelos próprios pacientes, que podem contribuir para o melhoramento dos serviços de saúde mental, como recomendado pela OMS.

\section{AGRADECIMENTOS}

À Secretaria Municipal de Saúde de Divinópolis, a toda a equipe do Serviço de Referência em Saúde Mental (Sersam) da referida cidade e a todos os pacientes que participaram, pelo apoio na realização desta pesquisa.

Este artigo faz parte de um projeto de pesquisa financiado pela Fundação de Amparo à Pesquisa do Estado de Minas Gerais (Fapemig), Conselho Nacional de Desenvolvimento Científico e Tecnológico (CNPq) e Universidade Federal de São João del-Rei (UFSJ).

Agências financiadoras: Fapemig, CNPq e UFSJ.

Conflito de interesses: nenhum.

\section{REFERÊNCIAS}

1. Bandeira M, Barroso SM. Sobrecarga das famílias de pacientes psiquiátricos. J Bras Psiquiatr. 2005;54(1):34-46.

2. Souza LA, Coutinho ESF. Fatores associados à qualidade de vida de pacientes com esquizofrenia. Rev Bras Psiq. 2006;28(1):50-8.

3. World Health Organization. WHO-SATIS Consumer's and Caregivers' satisfaction with Mental Health Services - A Multisite Study. Division of Mental Health. Geneva; 1996.

4. Organización Mundial de la Salud. Conjunto de guías sobre servicios y politicas de salud mental. Mejora de la calidad de la salud mental. Editores Médicos SA. Edimsa; 2003.

5. Pitta AMF. Qualidade de vida de clientes de serviços de saúde mental. Rev Psiq Clín. 1999;26(2):68-77.

6. Daltio CS, Mari JJ, Ferraz MB. Estudos farmacoeconômicos e carga da doença em esquizofrenia. Rev Psiq Clín. 2007;34(2):208-12.

7. Awad AG, Voruganti LNP, Heslegrave RJ. A conceptual model of quality of life in schizophrenia: description and preliminary clinical validation. Qual Life Res. 1997;6:21-6.

8. Russo J, Roy-Byrne P, Jaffe C, Ries R, Dagadakis C, O'Connor ED, et al. The relationship of patient-administered outcome assessments to quality of life an physician ratings: validity of the BASIS-32. J Ment Heath Adm. 1997;24(2):200-14.

9. Voruganti LNT, Heslegrave R, Awad AG, Seeman MV. Quality of life measurement in schizophrenia: reconciling the quest for subjectivity with the question of reliability. Psychol Med. 1998;28:165-72.
10. Simon-Abbadi S, Guelfi JD, Ginestet D. Psychometric qualities of the French version of the Heinrichs quality of life rating scale. Eur Psychiatry. 1999;14:386-91.

11. Cramer JA, Rosenheck R, Xu W, Thomas J, Henderson W, Charney DS. Quality of life in schizophrenia: a comparison of instruments. Schizophr Bull. 2000;26(3):659-66.

12. Wilkinson G, Hesdon B, Wild D, Cookson R, Farina C, Sharma V, et al. Self-report quality of life measure for people with schizophrenia: the SQLS. Br J Psychiatry. 2000;177:42-6.

13. Fitzgerald PB, Williams CL, Corteling N, Filia SL, Brewer K, Adams A, et al. Subject and observer-rated quality of life in schizophrenia. Acta Psychiatr Scand. 2001;103:387-92.

14. Gaite L, Vazquez-Barquero IL, Borra C, Ballesteros J, Schene A, Welcher B, et al. Quality of life in patients with schizophrenia in five European countries: the EPSILON study. Acta Psychiat Scand. 2002;105(4):283-92.

15. Ritsner M, Kurs R, Gibel A, Hirschmann S, Shinkarenko E, Ratner Y. Predictors of quality of life in major psychoses: a naturalistic follow-up study. J Clin Psychiatry. 2003;64:308-15.

16. Chan S, Yu lu W. Quality of life of clients with schizophrenia. J Adv Nurs. 2004;45(1):72-83.

17. Caron J, Lecomte W, Stip E, Renaud S. Predictors of quality of life in schizophrenia. Community Ment Health J. 2005;41(4):399-417.

18. Cavieres A, Valdebenito M. Funcionamiento cognitivo y calidad de vida en la esquizofrenia. Rev Chil Neuropsiquiatr. 2005;43(2):97-108.

19. United States Food and Drugs Administration. Guidance for Industry. Patient-Reported Outcome Measures: Use in Medical Product Development to Support Labeling Claims. FDA; 2006.

20. Mercier C, Landry M, Corbiere M, Perreault M. Measuring clients' perception as outcome measurement. In: Roberts AR, Yeager KR. Evidence-based Practice Manual: Research an Outcome Measures in Health and Human Services. Oxford: University Press; 2004, p. 904-9.

21. Selltiz C, Wrightsman LS, Cook SW. Métodos de pesquisa nas relações sociais. São Paulo: EPU; 1976.

22. Contandriopoulos AP, Champagne F, Potvin L, Denis JO, Boyle P. Saber preparar uma pesquisa. São Paulo: Hucitec Abrasco; 1994.

23. Organização Mundial de Saúde. CID-10: Classificação Internacional de Doenças e Problemas Relacionados à Saúde. Décima Revisão. São Paulo; 1998.

24. Cardoso CS, Caiaffa WT, Bandeira M, Siqueira AL, Abreu MNS, Fonseca JOP. Qualidades psicométricas da escala de qualidade de vida para pacientes com esquizofrenia: escala QLS-BR. J Bras Psiquiatr. 2003;52(3):211-22.

25. Bandeira M, Calzavara MGP, Costa SC, Cesari L. Avaliação dos serviços de saúde mental: adaptação transcultural de uma medida da percepção dos usuários sobre os resultados do tratamento. J Bras Psiquiatr. 2009:58(2):107-14.

26. Bandeira M, Andrade MCR, Costa CS, Silva MA. Percepção dos pacientes sobre o tratamento em serviços de saúde mental: validação da Escala de Mudança Percebida. Psicologia: Reflexão e Crítica. 2011. [no prelo]

27. Hosmer DW, Lemeshou S. Applied logistic regression. New York: John Wiley \& Sons; 2000.

28. Bisquerra R, Sarriera JC, Matínez F. Introdução à estatística: enfoque informático com 0 pacote estatístico SPSS. Porto Alegre: Artmed; 2004.

29. Montgomery DC, Peck EA. Introduction to linear regression analysis. 2. ed. New York: John Wiley \& Sons; 1992

30. Cesari L. Percepção de mudança e qualidade de vida em pacientes com esquizofrenia [dissertação]. São João del-Rei: Universidade Federal de São João del-Rei; 2010.

31. Santana AFFA, Chianca TCM, Cardoso CS. Qualidade de vida de pacientes com esquizofrenia internados em hospital de custódia. J Bras Psiquiatr. 2009;58(3):187-94.

32. Skantze K, Malm U, Dencker SJ, May PR, Corrigan P. Comparison of quality of life with standard of living in schizophrenic out-patients. Br J Psychiatry. 1992;161:797-801.

33. Priebe S, Warner R, Hubschmid T, Eckle I. Employment, attitudes toward work, and quality of life among people with schizophrenia in three countries. Schizophr Bull. 1998;24(3):469-77.

34. Costa SC. Percepção de mudança em função do tratamento recebido nos serviços de saúde mental: comparação entre pacientes e familiares [dissertação]. São João del-Rei: Universidade Federal de São João del-Rei; 2010

35. Perreault M, White ND, Fabrès E, Landry M, Anestin AS, Rabouin D. Relationship between perceived improvement and treatment satisfaction among clients of a methadone maintenance program. Evaluation and Program Planning. 2010. [in press] 